Pacific Journal of Mathematic 


\title{
A MAXIMUM PRINCIPLE ON CLIFFORD TORUS AND NON-EXISTENCE OF PROPER HOLOMORPHIC MAP FROM THE BALL TO POLYDISC
}

\author{
B. WONG
}

\begin{abstract}
A maximum principle which estimates the gradients on the clifford torus of plurisubharmonic functions defined in the polydisc is derived. With this result we give a new proof that there exists no proper holomorphic map from the ball to polydisc in $C^{2}$.
\end{abstract}

1. Introduction. It is an old theorem of $\mathrm{H}$. Poincare that the bidisc $A_{2}=\left\{\left(z_{1}, z_{2}\right):\left|z_{1}\right|<1,\left|z_{2}\right|<1\right\}$ and the ball $B_{2}=\left\{\left(z_{1}, z_{2}\right):\left|z_{1}\right|^{2}+\right.$ $\left.\left|z_{2}\right|^{2}<1\right\}$ are holomorphically distinct. Around 1935, V. Rothstein proved the remarkable fact that there exists no proper holomorphic map from $A_{2}$ to $B_{2}$ ([6]). Much later, G. H. Henkin generalised this result of Poincaré to the care of analytic polyhedron and strictly pseudo-convex domain ([3]). His proof was based on the comparison of Carathéodory metrics on these two different domains. He also indicated that it is possible to yield a generalization of Rothstein's theorem to polyhedron and strictly pseudo-convex domain by using the techniques employed in his paper (i.e. there exists no proper holomorphic map from polyhedron to strictly pseudo-convex domain). But his method can only be applied to the case of proper holomorphic map from polyhedron to strictly pseudo-convex domain. Despite all these old and new developments, a proof the following statement is unknown to the author.

"There exists no proper holomorphic map from $B_{2}$ to $A_{2}$ ".

The proofs of Poincaré and Rothstein are rather easy; they used the groups of biholomorphisms, Carathéodory metrics as well as the continuity principle to gain contradiction. In order to obtain a proof of the above statement a deeper investigation is in demand. In this paper we give a proof of this fact along the line of the theory of intrinsic measures (Carathéodory measures, Eisenman-Kobayashi measures) ([4], [5], [7]). The proof involves a boundary estimate of Eisenman-Kobayashi measure in $A_{2}-W$, where $W$ is a complex analytic variety.

For simplicity of notations we shall restrict all our statements in $C^{2}$, but our proof can equally be applied to $C^{n}$ without difficulty. One can also generalize our result to strictly pseudo-convex domain by passing through a localization process. It is also important to point out that, throughout this paper, the intrinsic measures are defined with respect to polydisc ([5]). They are different, in an 
essential way, from those measures defined with respect to the ball ([7]). For the basic theory of intrinsic measures one should consult [4] and [5]. They will be used frequently in the sequel of this paper.

After the completion of our work we were informed by professors Yum-Tong Siu and Karl Stein that our result had already been proved by H. Rischel (Math. Scand. 15 (1964), 49-63). Our proof is entirely different from his, and the method of invariant measures can be used to prove further interesting results on proper holomorphic maps between strongly pseudo-convex domains in $\boldsymbol{C}^{n}$. Although there are difficulties for us to understand the proof due to Rischel, it should be stressed that his paper has been available for fifteen years and our proof was obtained independently.

1. Maximum principle on clifford torus. The distinguished boundary of $\Delta_{2}$, namely

$$
T=\left\{\left(z_{1}, z_{2}\right)|| z_{1} \mid=1 \text { and }\left|z_{2}\right|=1\right\},
$$

can be regarded as a regularly embedded torus in

$$
S^{3}=\left\{\left.\left(z_{1}, z_{2}\right)|| z_{1}\right|^{2}+\left|z_{2}\right|^{2}=2\right\},
$$

which is a three dimensional sphere. In the terms of classical differential geometry, $T$ is called a clifford torus. The clifford torus plays an important role in the complex function theory of polydisc. On the other hand, it is an interesting submanifold of $S^{3}$ from a geometric viewpoint.

Let $x$ be a point of $T \subset S^{3}$. We shall use the following notations throughout;

(1) $n_{x}$ is the line pointing from the origin of $S^{3}$ to $x$ (i.e., radial direction).

(2) If $z \in n_{x}(x \in T)$ we denote by $d(z, T)$ the euclidean distance from $z$ to $T$.

(3) $N_{\varepsilon}=\left\{z \in \Delta_{2} \mid z \in n_{t}, t \in T, d(z, T)<\varepsilon\right\}$, were $\varepsilon$ is a sufficiently small positive number.

We are going to derive the following maximum principle in this paragraph.

THEOREM 1.1. Let $U$ be a funciton in $\Delta_{2}$ which is continuous up to the boundary of $\Delta_{2}$. We assume that

(1) $U \equiv 0$ on $\partial \Delta_{2}$ and $U<0$ in $\Delta_{2}$.

(2) $U$ is plurisubharmonic in $\Delta_{2}$.

Then there exists a small $\varepsilon>0$ and a positive constant $C$ such 
that for all $z \in N_{\varepsilon},-U(z) / d(z, T) \geqq C>0$.

Proof. Let us write

$$
g(z)=e^{-a r^{2}}-e^{-2 a},
$$

where $z=\left(z_{1}, z_{2}\right) \in C^{2}, a$ is a constant to be determined, $r=|z|=$ $\sqrt{\left|z_{1}\right|^{2}+\left|z_{2}\right|^{2}}$.

A direct computation would show that $g$ satisfies the following conditions

(1) $\forall z \in \Delta_{2}, g(z)>0$

(2) $\forall z \in T, g(z) \equiv 0$

(3) There exists a positive constant $K$ such that $d g / d n_{t}(t)=$ $-2 \sqrt{2} a e^{-2 a}<-k$ for all $t \in T$.

Let $r_{1}$ be a positive number which is slightly less that $\sqrt{2}$. Then $\partial B_{2}^{r_{1}}=\left\{\left.\left(z_{1}, z_{2}\right)|| z_{1}\right|^{2}+\left|x_{2}\right|^{2}=r_{1}^{2}\right\}$. We also denote by $D_{1}$ the region between $\partial B_{2}^{\sqrt{2}}$ and $\partial B_{2}^{r_{1}}$ and $D=D_{1} \cap \Delta_{2}$. Let $A=D \cap$ $\left(\bigcup_{x \in T} \bar{n}_{x}\right)$, where $\bar{n}_{x}=\{\lambda x \mid \lambda$ is a complex number $|\lambda|<1, x \in T\}$.

The following is a result subject to only straightforward computation.

(4) If $a$ is a sufficiently large positive constant, then $\forall z \in A$, $\Delta g(z)>0$, where $\Delta$ is the Laplacian operator on $\bar{n}_{x}$ which contains $z$ with $x \in T$.

Now we can proceed with our proof.

Let $V=U+s \cdot g$, where $s$ is a positive constant such that $V(z)<0$ for all $z$ belonging to $\partial A \cap \partial B_{2}^{r_{1}}$. It should be noticed that the sets $T$ and $\partial A \cap \partial B_{2}^{r_{1}}$ constitute the boundary of $A$ constructed above.

Claim. If we regard $V$ as a function defined on $A$, then it is impossible for $V$ to attain its maximum in the interior of $A$.

Suppose $w \in A$ such that $V(w)$ is the maximum. Let $\bar{n}_{x}$ be the complex disc as above which contains $w$ (where $x \in T$ ). However, $U$ is plurisubharmonic in $A_{2}$ (therefore subharmonic on $\bar{n}_{x}$ ) and $g$ is subharmonic on $\bar{n}_{x}$, one can easily see that $V=U+s \cdot g$ is then subharmonic on $\bar{n}_{x}$. Thus one obtains a contradiction to the assumption that $V$ attains a maximum at $w$. Furthermore $V(z)<0$ for all $z \in \partial A \cap \partial B_{2}^{r_{1}}$, hence it is led to the conclusion that $V$ attains its maximum on $T$; here we have used the fact that $g=0, U=0$ on $T$ and that both $g$ and $U$ are continuous up to the boundary.

By the compactness of $T$ and the fact that the vector field $n_{t}$ on $T$ is smooth we can choose a sufficiently small $\varepsilon>0$ such that

$$
\frac{U(t)-U(z)}{d(z, \partial T)}+s \cdot \frac{d g}{d n_{x}}(t) \geqq 0
$$

for all $t \in T, z \in N_{\varepsilon}$. 
Since $U(t) \equiv 0 \forall t \in T$, together with condition (3) we obtain

$$
-\frac{U(z)}{d(z, T)} \geqq-s \cdot \frac{d g}{d n_{t}} \geqq s \cdot K>0
$$

for all $t \in T, z \in N_{\varepsilon}$.

Letting $C=s \cdot K$ we complete the proof.

2. A theorem on the proper holomorphic map from $B_{2}$ to $\Delta_{2}$. Suppose $f: B_{2} \rightarrow \Delta_{2}$ is a proper holomorphic map. By a theorem of Remmert $\left(B_{2}, f, A_{2}\right)$ is a complex analytic branched covering. The branching locus is given by $s=\left\{z \in B_{2} \mid \operatorname{det}(d f(z))=0\right\}$ and $f: B_{2}-s \rightarrow$ $\Delta_{2}-f(s)$ is a unramified finite complex analytic covering.

We define a complex analytic function $L: A_{2}-f(s) \rightarrow C$ as follows,

$$
L(z)=\prod_{i=1}^{m} \operatorname{det}\left(d f\left(x_{i}\right)\right), \quad \text { where } \quad x \in \Delta_{2}-f(s),
$$

$f^{-1}(z)=\left\{x_{1}, x_{2}, \cdots, x_{m}\right\}, d f\left(x_{i}\right)=$ Jacobian of $f$ at $x_{i}$. We make an observation here that $L$ is locally bounded around the complex subvariety $f(s) \subset \Delta_{2}$, hence $L$ extends holomorphically across $f(s)$. The following theorem will be proved in this section.

THEOREM 2.1. L is a bounded holomorphic function in $\Delta_{2}$.

Proof. Let $r$ be a positive number slightly less than 1 . It is well-known fact that $L \mid \Delta_{2}^{r}$ assumes its maximum on the distinguished boundary

$$
T\left(\Delta_{2}^{r}\right)=\left\{\left(z_{1}, z_{2}\right)|| z_{1}|=r,| z_{2} \mid=r\right\}
$$

of $\Delta_{2}^{r}$.

With some considerations it suffies to prove that $\forall z \in N_{\varepsilon}$, $|\operatorname{det}(d f(x))| \leqq Q$, where $x \in\left\{f^{-1}(x)\right\}, Q$ is a constant and $\varepsilon$ is a sufficiently small positive number.

The following notations will be used in the rest of this paper: $E_{B_{2}}=$ Eisenman-Kobayashi measure on $B_{2}$

$E_{B_{2}}^{\sqrt{2}}=$ Eisenman-Kobayashi measure on $B_{2}^{\sqrt{2}}=\left\{\left.\left(z_{1}, z_{2}\right)|| z_{1}\right|^{2}+\left|z_{2}\right|^{2}<2\right\}$ $E_{\Delta_{2}}=$ Eisenman-Kobayashi measure on $\Delta_{2}$

(For the definition of Eisenman-Kobayashi measure, consult [4]). The following fact are immediate

(a) Since $f: B_{2} \rightarrow \Delta_{2} \subset B_{2}^{\sqrt{2}}$ is holomorphic map, by the measuredecreasing property we have

$$
\left|E_{B_{2}}(x)\right| \geqq|\operatorname{det}(d f(x))| \cdot\left|E_{B_{2}}^{\sqrt{2}}(z)\right|
$$

where 


$$
x \in\left\{f^{-1}(z)\right\}, \quad z \in \Delta_{2} \subset B_{2}^{\sqrt{2}} .
$$

(b) From the explicit formulas of $E_{B_{2}}$ and $E_{B_{2}}^{\sqrt{2}}$, we have

$$
\begin{gathered}
\frac{C_{1}}{\left(\left(y, \partial B_{2}\right)\right)^{3}} \leqq\left|E_{B_{2}}(y)\right| \leqq \frac{C_{2}}{\left(\left(y, \partial B_{2}\right)\right)^{3}} \\
\frac{K_{1}}{\left(d\left(w, \partial B_{2}^{\sqrt{2}}\right)\right)^{3}} \leqq\left|E_{B_{2}}{ }^{\sqrt{2}}(w)\right| \leqq \frac{K_{2}}{\left(d\left(w, \partial B_{2}^{\sqrt{2}}\right)\right)^{3}}
\end{gathered}
$$

where $y \in B_{2}, w \in B_{2}^{\sqrt{2}}$, sufficiently close to the boundary, $C_{1}, C_{2}, K_{1}$ and $K_{2}$ are constants.

(c) From the explicit formulas of Kobayashi metrics in $B_{2}$ and $\Delta_{2}$, and also the distance-decreasing property, we have

$$
d\left(z, \partial \Delta_{2}\right) \geqq l \cdot d\left(x, \partial B_{2}\right),
$$

where $x \in\left\{f^{-1}(z)\right\}$ for all $z \in \Delta_{2}$ sufficiently close to $\partial \Delta_{2}, l$ is a positive constant.

With the above facts in mind we can prove the following consequences.

$$
\left(\frac{C_{2}}{K_{2}}\right) \cdot\left(\frac{d\left(z, \partial B_{2}^{\sqrt{2}}\right)}{d\left(x, \partial B_{2}\right)}\right)^{3} \geqq|\operatorname{det}(d f(x))|
$$

with $x \in\left\{f^{-1}(z)\right\}, z \in N_{\varepsilon}, \varepsilon$ is a sufficiently small number.

(2) Suppose that $U: A_{2} \rightarrow R$ is defined as follows;

$$
\begin{aligned}
U(z)= & \max \left\{-d\left(x_{1}, \partial B_{2}\right),-d\left(x_{2}, \partial B_{2}\right), \cdots,\right. \\
& \left.-d\left(x_{m}, \partial B_{2}\right) \mid f^{-1}(z)=\left\{x_{1}, x_{2}, \cdots, x_{m}\right\}, z \in \Delta_{2}\right\} .
\end{aligned}
$$

Obviously $U$ is a bounded plurisubharmonic function on $\Delta_{2}-$ $\{w \mid L(w)=0\}$, it extends across $\{w \mid L(w)=0\}$ by a well-known lemma of Grauert. By (c) it is easy to see that $U$ is continuous up to the boundary of $\Delta_{2}$.

Finally we apply Theorem 1.1 to $U$ defined above. We therefore obtain

$$
\frac{1}{C} \geqq \frac{d\left(z, \partial B_{2}^{\sqrt{2}}\right)}{d\left(x, \partial B_{2}\right)} \quad(C \text { is the constant in Theorem 1.1) },
$$

where $x \in\left\{f^{-1}(z)\right\}, z \in N_{\varepsilon}$ for a well-chosen small positive constant $\varepsilon$ (Note: if $z \in N_{\varepsilon}, d\left(z, \partial B_{2}^{\sqrt{2}}\right)=d(z, T)$.)

Combining all above inequalities, we have

$$
\left(\frac{1}{C}\right)^{3}\left(\frac{C_{2}}{K_{1}}\right) \geqq|\operatorname{det}(f(x))| \text {. }
$$

The proof is thereby completed. 
3. Boundary behavior of the complex analytic variety $\left\{z \in \Delta_{2} \mid L(z)=0\right\}$ in $\Delta_{2}$. Let $P$ be a point belonging to $\partial \Delta_{1}$. We denote by $\Gamma_{P}^{\theta}$ the cone extending $\theta$ with vertex at $P$ in $\Delta_{1}$ (i.e., $\Gamma_{P}^{\theta}=$ $\left.\left\{z \mid z=(x, y), z \in \Delta_{1}, \tan ^{-1}(y / x)<\theta, 0 \leqq \theta<\pi / 2\right\}\right)$, where we choose $P$ to be the origin of our coordinates, $x$ - axis $=$ normal of $\partial \Delta_{1}$ at $P$, $y$ - axis = tangent of $\partial \Delta_{1}$ at $P$. Furthermore, $P_{1}: \Delta_{1} \times \Delta_{1} \rightarrow \Delta_{1}$, $P_{2}: \Delta_{1} \times \Delta_{1} \rightarrow \Delta_{1}$ are first and second projections in a natural way.

LEMMA 3.1. With the same notations as before, there exists a point $P \in T\left(\Delta_{2}\right)$ such that it is not an accumulation point of $\{z \mid L(z)=0\} \cap \Lambda_{P}^{\theta}$ for all $0 \leqq \theta<\pi / 2$, where $\Lambda_{P}^{\theta}=\Gamma_{P}^{\theta} \times \Gamma_{P}^{\theta} \subset \Delta_{2}$.

Proof. By Theorem 2.1. $L$ is a bounded holomorphic function, the radial limits $L^{*}$ of $L$ approaching $T\left(\Delta_{2}\right)$ exist almost everywhere. It is well-known fact that $L=\bar{P}\left[L^{*}\right]$ (see our remark below), where $\bar{P}$ is the poisson kernel of $\Delta_{2}$ (for instance, see Rudin: Function theory in polydiscs P. 31 exercise). Furthermore one can model from the proof of Fatou theorem to give the following assertion: For almost every point $y \in T\left(\Delta_{2}\right)$, the non-tangential limit

$$
\lim _{\substack{x \rightarrow y_{\theta} \\ x \in y_{y}}} L(x)
$$

exists, i.e.,

$$
L^{*}(y)=\lim _{\substack{x \rightarrow y_{\theta} \\ x \in y_{y}}} L(x)
$$

for $y \in T\left(A_{2}\right)$ a.e. However, if every point $y \in T\left(\Delta_{2}\right)$ is an accumulation of

$$
\{z \mid L(z)=0\} \cap \Lambda_{P}^{\theta} \quad \text { for some } \quad 0 \leqq \theta<\frac{\pi}{2},
$$

it would imply immediately that $L^{*}(y)=0$ a.e., hence $L \equiv 0$ by poisson formula. It is a contradiction.

REMark. To be rigorus we should write $L=L_{1}+i L_{2}$, where $L_{1}$ and $L_{2}$ are $n$-harmonic (see Rudin, p. 16), correspondingly $L^{*}=$ $L_{1}^{*}+i L_{2}^{*}$, and notation $L=\bar{P}\left[L^{*}\right]$ means $L_{1}=\bar{P}\left[L_{1}\right]$ and $L_{2}=\bar{P}\left[L_{2}^{*}\right]$.

The proof of our claim concerning the boundary values of bounded holomorphic functions on $T\left(A_{2}\right)$ is rather long; it is a reproduction of a theorem of Fatou which is a folklore in the area of boundary values of holomorphic functions, we therefore take the liberty to skip the proof here. Proofs and references of relevant results can be found in "A. Koranyi-E. M. Stein: Fatou's theorem for generalized half planes". (Estratto dagli Annali della, Scuola 
Normale Superiore di Pisa classe di Science, Vol. XXII, Fasc. I, (1968).)

4. An estimate of Eisenman-Kobayashi measure. We start with the definitions of Carathéodory and Eisenman-Kobayashi measures. It is easy to check the measure $U$ on $A_{2}$ given below is invariant under biholomorphisms;

$$
U=\prod_{j=1}^{2} \frac{4 \sqrt{-1}}{\left(1-\left|z^{j}\right|^{2}\right)^{2}} d z^{j} \Lambda d \bar{z}^{j} .
$$

(Note: We use the notation $|U|$ to stand for $\Pi_{j=1}^{2}\left(4 /\left|\left(1-\left|z^{j}\right|^{2}\right)^{2}\right|\right)$.)

Let $D$ be a bounded domain in $C^{2}$. The Carathéodory measure $C_{D}$ is defined as follows:

$$
C_{D}(x)=\sup _{f}\left(f^{*} U\right)_{x},
$$

where the supremun is taken over all holomorphic maps $f: D \rightarrow \Delta_{2}$.

The Eisenman-Kobayashi $E_{D}$ is defined as follows:

$$
E_{D}(x)=\inf _{f}\left(\left(f^{-1}\right) * U\right)_{0} .
$$

Where the infimun is taken over all holomorphic maps $f: \Delta_{2} \rightarrow D$ which maps the origin " $O$ " of $\Delta_{2}$ to $x$ and is nondegenerate at " $O$ ".

In this section we shall derive an estimate of Eisenman-Kobayashi measure (in the case of metric, such an estimate was first obtained by R. L. Royden).

Definition. $z, w \in D$, then $d_{D}(z, w)=\inf \left\{P(a, b) \mid f \in \operatorname{Hol}\left(\Delta_{2}, D\right)\right.$ s.t. $f(a)=z, f(b)=w, P$ is the Kobayashi metric in $\left.\Delta_{2}\right\}$.

Let $D_{1}$ be another domain in $C^{2}$ such that $D_{1} \cap D$ is nonempty.

Definition. For $z \in D \cap D_{1}$

$$
d_{D-D_{1}}(z)=\inf \left\{d_{D}(z, w) \mid w \text { belongs to } D \text { but not } D_{1}\right\} .
$$

Theorem 4.1. Let $\bar{D}=D \cap D_{1}$. Then for all $z \in \bar{D}$, we have

$$
\left|E_{\bar{D}}(z)\right| \leqq\left|\cot h d_{D-D_{1}}(z)\right|^{4} \cdot\left|E_{D}(z)\right| \text {. }
$$

Proof. First of all let us fix a constant $r$ as follows, $r=$ $\sup \left\{t \mid\right.$ there exists $\left.f \in \operatorname{Hol}\left(\Delta_{2}^{t}, \bar{D}\right), f(0)=z,|\operatorname{det}(d f(0))|=16\right\}$.

Then we choose a number $R$ slightly larger than $r$. From our choice of $r$ it is obvious that there is a $f \in \operatorname{Hol}\left(\Delta_{2}^{R}, D\right)$ s.t. $f(0)=z$, $|\operatorname{det}(d f(0))|=16$, and it maps a boundary point of $\Delta_{2}^{r}$ to a point belonging to $D-\bar{D}$. One can see, if $w$ is this point belonging to 
$D-\bar{D}$, then $d_{D-D_{1}}(z) \leqq d_{D}(z, w)$.

From the definition of $d_{D}(z, w)$ we observe that

$$
d_{D}(z, w) \leqq \frac{1}{2} \ln \left(\frac{1+\frac{r}{R}}{1-\frac{r}{R}}\right) .
$$

Then it implies

$$
\begin{aligned}
& \left(\frac{1}{r}\right) \leqq\left(\operatorname{coth} d_{D-D_{1}}(z)\right) \cdot\left(\frac{1}{R}\right) \\
& \left(\frac{1}{r}\right)^{4} \leqq\left(\operatorname{coth} h d_{D-D_{1}}(z)\right)^{4} \cdot\left(\frac{1}{R}\right)^{4} .
\end{aligned}
$$

It is easy to see that the above inequality together with the definition of Eisenman-Kobayashi measure imply our theorem immediately.

5. Some estimates of Kobayashi metric inside the cones. Let $D$ be a domain in $C^{n}$, we denote by $d_{D}^{k}$ the Kobayashi metric on $D$. If $D$ is the upper-half plane $H=\{x+i y \mid y>0\}, d_{H}^{k}$ is then induced by $\left(2 \sqrt{d x^{2}+d y^{2}}\right) / y$. Suppose $P$ is the origin of $z$-plane and $\Gamma_{P}^{\theta}$ is a cone in $H$ with vertex at $P$ and extended angle $\theta$, where $0 \leqq \theta<\pi / 2$

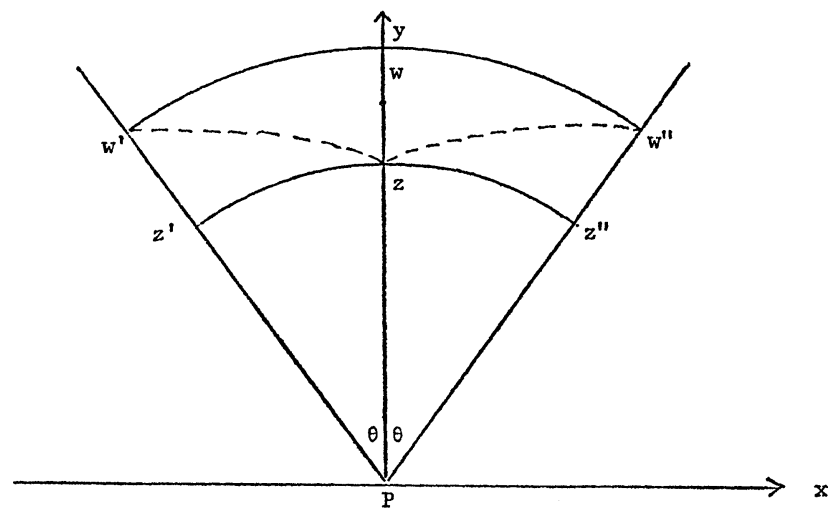

In the following theorem we assume that $z=(0, y)$ is a point on the $y$-axis.

THEOREM 5.1. $d_{H}^{k}\left(z, \partial \Gamma_{P}^{\theta}\right)=2 \ln (\tan \theta+\sec \theta)$ where $d_{H}^{k}\left(z, \partial \Gamma_{P}^{\theta}\right)=$ the distance from $z$ to the boundary of $\Gamma_{P}^{\theta}$ (i.e., $\partial \Gamma_{P}^{\theta}$ ) with respect to $d_{H}^{k}$.

Proof. It is well-known that the geodesic of the metric 
$\left(2 \sqrt{d x^{2}+d y^{2}}\right) / y$ passing through $z$ is a great circle (with radius $|y|$ and center at $P$ ). Let $z^{\prime}$ and $z^{\prime \prime}$ be the points of intersection of this circle with $\partial \Gamma_{P}^{\theta}$. Then the length of the arc $z z^{\prime}$ is equal to

$$
2 \int_{0}^{\theta} \frac{y d \phi}{(y \cos \phi)}=2 \int_{0}^{\theta} \frac{d \phi}{\cos \phi}=2 \ln (\tan \theta+\sec \theta) .
$$

We need the following known result in Riemannian geometry for our proof.

THeOREM. Let $M$ be a simply connected complete Riemannian manifold of negative sectional curvature, then for an two points in $M$ there exists one and only one minimizing geodesic joining them.

Now suppose that $w^{\prime}$ is a point on $\partial \Gamma_{P}^{\theta}$ such that the length of $z w^{\prime}=d_{H}^{k}\left(z, \partial \Gamma_{P}^{\theta}\right)$. By the symmetric properties of $\Gamma_{P}^{\theta}$ and $\left(2 \sqrt{d x^{2}+d y^{2}}\right) / y$ one easily observes that there exists a point $w^{\prime \prime}$ on the other side of $\Gamma_{P}^{\theta}$ s.t. $P w^{\prime \prime}=P w^{\prime}$ (with respect to euclidean length). Let $w$ be the point of intersection of $y$-axis and the great circle of radius $P w^{\prime}$ and with center at $P$. Since the arc $w w^{\prime}$ is a minimizing geodesic joining $w$ and $w^{\prime}$, hence $d_{H}^{k}\left(w, w^{\prime}\right) \leqq d_{H}^{k}\left(z, w^{\prime}\right) . d_{H}^{k}\left(w, w^{\prime}\right)=d_{H}^{k}\left(z, z^{\prime}\right)=2 \ln (\tan \theta+\sec \theta)$ from the previous computation. Thus, one obtains

$$
d_{H}^{k}\left(z, z^{\prime}\right) \leqq d_{H}^{k}\left(z, w^{\prime}\right)=d_{H}^{k}\left(z, \partial \Gamma_{P}^{\theta}\right) .
$$

It is now easy to conclude that

$$
d_{H}^{k}\left(z, \partial_{P}^{\theta} \Gamma\right)=2 \ln (\tan \theta+\sec \theta) .
$$

THEOREM 5.2. Let $P$ be a boundary point of the unit disc $\Delta_{1}$. Suppose that $\Gamma_{P}^{\theta}$ is a cone in $\Delta_{1}$ with vertex at $P$ and extended angle $\theta, 0 \leqq \theta<\pi / 2, z$ is a point of the normal of $\partial \Delta_{1}$ at $P$. (See our. figure.) Then we have $d_{\Delta_{1}}^{k}\left(z, \partial \Gamma_{P}^{\theta}\right) \geqq 2 \ln (\tan \theta+\sec \theta)$.

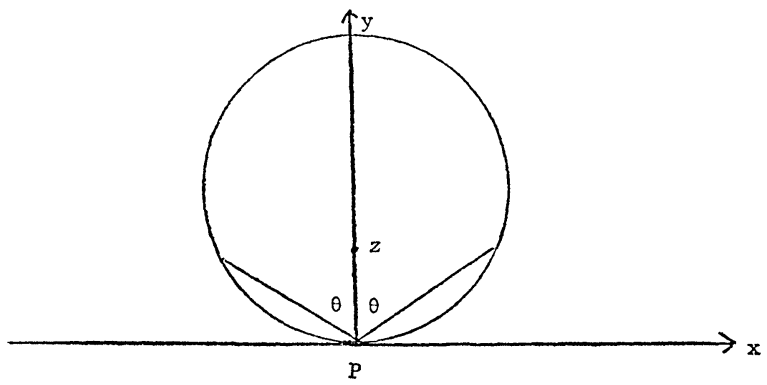

Proof. Since $H \supset \Delta_{1}$, by the distance-decreasing property of Kobayashi metrics we have 


$$
d_{\Delta_{1}}^{k}\left(z, \partial \Gamma_{P}^{\theta}\right) \geqq d_{H}^{k}\left(z, \partial \Gamma_{P}^{\theta}\right) .
$$

Our theorem follows immediately from Theorem 5.1.

THEOREM 5.3. Let $P$ be a point on $T\left(\Delta_{2}\right)$ and $\Lambda_{P}^{\theta}$ a product cone as before, $0 \leqq \theta<\pi / 2$. Then we have

$$
d_{\Delta_{2}}^{k}\left(z, \partial \Lambda_{P}^{\theta}\right) \geqq 2 \ln (\tan \theta+\sec \theta) .
$$

Proof. It is clear from Theorem 5.2 and the remark below.

REMARK. It is a well-known fact in the theory of intrinsic metrics that

$$
d_{\Delta_{2}}^{k}\left(z_{1}, z_{2}\right)=\sup \left\{d_{\Delta_{1}}^{k}\left(P_{1}\left(z_{1}\right), P_{1}\left(z_{2}\right)\right), d_{\Delta_{1}}^{k}\left(P_{2}\left(z_{1}\right), P_{2}\left(z_{2}\right)\right)\right\} .
$$

It is now easy to observe the following.

THEOREM 5.4. With the assumptions in Theorem 5.3, we have

(1) $\operatorname{Lim}_{\theta \rightarrow \pi / 2} d_{\Delta_{2}}^{k}\left(z, \partial \Lambda_{P}^{\theta}\right)=\infty$

(2) $\operatorname{Lim}_{\theta \rightarrow \pi / 2} \cot h d_{\Delta_{2}}^{k}\left(z, \partial \Lambda_{P}^{\theta}\right)=1$.

Proof. Elementary.

6. Main part of the proof. We break our proof into several steps:

(1) From Lemma 3.1 there exists a point, namely $p \in T\left(\Delta_{2}\right)$, which is not an accumulation point of $f(s) \cap \Lambda_{P}^{\theta}$ in $\Lambda_{P}^{\theta}$ for all $0 \leqq$ $\theta<\pi / 2$. We choose a sequence $\left\{z_{i}\right\} \rightarrow P$, where $z_{i}$ belongs to $A_{2}-f(s)$ and lies on the line $R$ perpendicular to $S^{3}$ at $P$. Let $\left\{x_{i}\right\}$ be another sequence in $B_{2}$, where $x_{i} \in\left\{f^{-1}\left(z_{i}\right)\right\}$. One easily verifies that $\left\{x_{i}\right\} \rightarrow q$ (passing through a subsequence if necessary), where $q$ is a boundary point $B_{2}$.

(2) $f: B_{2} \rightarrow \Delta_{2}$ is a proper holomorphic map. Then

$$
s=\left\{z \in B_{2} \mid \operatorname{det}(d f(z))=0\right\}
$$

and $f(s)$ are complex analytic varieties in $B_{2}$ and $A_{2}$ respectively. Furthermore, $f: B_{2}-s \rightarrow \Delta_{2}-f(s)$ is a finite complex analytic covering. From the standard facts in theory of intrinsic measures ([4], [5]) we have

(I ) $C_{A_{2}-f(s)}=C_{A_{2}}, C_{B_{2}-s}=C_{B_{2}}$

(II) $E_{\Delta_{2}-f(s)} \geqq E_{\Delta_{2}}, E_{B_{2}-s} \geqq E_{B_{2}}$

(III) $\quad C_{B_{2}} \geqq f^{*}\left(C_{\Delta_{2}}\right)$ $E_{B_{2}} \geqq f^{*}\left(E_{A_{2}}\right)$.

(Volume-decreasing property under holomorphic maps.) 
(IV) $E_{D} \geqq C_{D}$, where $D$ is any bounded domain

(V) $E_{B_{2}-s}=f^{*}\left(E_{\Delta_{2}-f(s)}\right)$

(note: $\left(B_{2}-s, f, A_{2}-f(s)\right.$ ) is a covering).

(3) Making use of the above inequalities and equalities we obtain

$$
1 \geqq \frac{\left|C_{B_{2}}\left(x_{i}\right)\right|}{\left|E_{B_{2}}\left(x_{i}\right)\right|} \geqq \frac{\left|f^{*}\left(C_{\Delta_{2}}\left(z_{i}\right)\right)\right|}{\left|E_{B_{2}-s}\left(x_{i}\right)\right|}=\frac{\left|C_{\Delta_{2}}\left(z_{i}\right)\right|}{\left|E_{\Delta_{2}-f(s)}\left(z_{i}\right)\right|}=\frac{\left|E_{\Delta_{2}}\left(z_{i}\right)\right|}{\left|E_{\Delta_{2}-f(s)}\left(z_{i}\right)\right|},
$$

for all $i$.

(4) The following lemma was derived in ([7]).

LEMMA. Let $D$ be a complete hyperbolic bounded domain in $C^{n}$. Suppose that $E_{D}$ and $C_{D}$ are defined with respect to polydisc $\Delta_{n}$. If there exists $x \in D$ such that

$$
\frac{\left|E_{D}(x)\right|}{\left|C_{D}(x)\right|}=1
$$

then $D$ is biholomorphic to $\Delta_{n}$.

Since $B_{2}$ is homogeneous we have

$$
\frac{\left|C_{B_{2}}\left(x_{i}\right)\right|}{\left|E_{B_{2}}\left(x_{i}\right)\right|}=C, \quad \text { for all } i,
$$

where $C$ is a constant which is not equal to 1 . It is clear from (3) that we would obtain a contradiction if the following identity holds

$$
\lim _{z_{i} \rightarrow P} \frac{\left|E_{\Delta_{2}}\left(z_{i}\right)\right|}{\left|E_{\Delta_{2}-s}\left(z_{i}\right)\right|}=1
$$

(5) First of all we know that

$$
E_{\Delta_{2}-f(s)} \geqq E_{\Delta_{2}} \quad \text { from (2) (II) . }
$$

Secondly, in the view of Theorem 4.1 we have the following inequality

$$
\left|E_{\Delta_{2}-f(s)}\left(z_{i}\right)\right| \leqq\left|\cot h d_{D-D_{1}}\left(z_{i}\right)\right|^{4} \cdot\left|E_{\Delta_{2}}\left(z_{i}\right)\right|
$$

where $D=\Delta_{2}, D_{1}=\Delta_{2}-f(s)$. Our proof would be completed if one could show

$$
\operatorname{Lim}_{z_{i} \rightarrow P} \cot h d_{D-D_{1}}\left(z_{i}\right)=1 .
$$

We note that $d_{D-D_{1}}\left(z_{i}\right)=d_{\Delta_{2}}\left(z_{i}, f(s)\right)$, where $d_{\Delta_{2}}$ was defined in $\S 4$. It is trivial to observe $d_{\Delta_{2}}=d_{\Delta_{2}}^{k}$ from our definition. Thus it is enough for us to prove 


$$
\operatorname{Lim}_{z_{i} \rightarrow P} \cot h d_{\lrcorner_{2}}^{k}\left(z_{i}, f(s)\right)=1
$$

(i.e., $\lim _{z_{i} \rightarrow P} d_{\Delta_{2}}^{k}\left(z_{i}, f(s)\right)=\infty$ ).

From our assumption $P$ is not an accumulation point of $f(s) \cap \Lambda_{P}^{\theta}$ in $\Lambda_{P}^{\theta}$ for all $0 \leqq \theta<\pi / 2$, for a fixed $\theta$ between 0 and $\pi / 2$ we have

$$
d_{\Delta_{2}}^{k}\left(z_{i}, f(s)\right) \geqq d_{\Delta_{2}}^{k}\left(z, \partial \Lambda_{P}^{\theta}\right)
$$

if $i$ is sufficiently large. However, it follows from Theorem 5.4 that

$$
d_{\Delta_{2}}^{k}\left(z_{i}, \partial \Lambda_{P}^{\theta}\right) \geqq 2 \ln (\tan \theta+\sec \theta) .
$$

Now we can conclude

$$
\operatorname{Lim}_{i \rightarrow \infty} d_{d_{2}}^{k}\left(z_{i}, f(s)\right) \geqq 2 \ln (\tan \theta+\sec \theta)
$$

for a fixed $\theta$.

Letting $\theta \rightarrow \pi / 2$, we thereby complete our proof (Theorem 5.4).

\section{REFERENCES}

1. L. Bers, F. John and M. Schechter, Partial differetial equations, Lectures in Applied Mathematics, Vol. 3 Interscience, N. Y., 1966.

2. S. S. Chern, Some open problems on complex manifolds, (paper presented to Amer. Math. Soc. Proceedings of Symposia in Pure Mathematics. "Mathematical problems arising from Hilbert").

3. G. H. Henkin, An analytic polyhedron is not holomorphically equivalent to a strictly pseudoconvex domain, Dokl. Acad. Nauk SSR Tom 210 (1973), No. 5 Soviet Mathematics Dokl. Vol. 14 (1973), Vol. 3.

4. S. Kobayashi, Hyperbolic Manifolds and Holomorphic Mappings, Kekker, N. Y., 1970.

5. - Intrinsic distance, measures and geometric function theory, Amer. Math. Soc. Bull., 82 (1976), 357-416.

6. R. Remmert and K. Stein, Eigentliche holomorphe, Abbildungen, Mathematics, Z 73 (1960), 159-189.

7. B. Wong, Characterization of the unit ball by its automorphism group, Inventiones Mathematicae, 41 (1977), 253-257.

Received July 25, 1978.

The Chinese University of Hong Kong

Shatin, N. T., Hong Kong 


\section{PACIFIC JOURNAL OF MATHEMATICS}

\section{EDITORS}

DONALD BABBITT (Managing Editor)

University of Galifornia

Los Angeles, California 90024

HUGo RossI

University of Utah

Salt Lake City, UT 84112

C. C. MOORE AND ANDREW OGG

University of California

Berkeley, CA 94720

\section{J. DUGUNDJI}

Department of Mathematics University of Southern California Los Angeles, California 90007

R. FINN AND J. MILGRAM Stanford University Stanford, California 94305

ASSOCIATE EDITORS
E. F. BECKENBACH
B. H. NeumanN
F. WoLF
K. YosHIDA

\section{SUPPORTING INSTITUTIONS}

UNIVERSITY OF BRITISH COLUMBIA UNIVERSITY OF SOUTHERN CALIFONIA CALIFORNIA INSTITUTE OF TECHNOLOGY UNIVERSITY OF CALIFORNIA MONTANA STATE UNIVERSITY STANFORD UNIVERSITY UNIVERSITY OF HAWAII UNIVERSITY OF TOKYO UNIVERSITY OF NEVADA, RENO UNIVERSITY OF UTAH NEW MEXICO STATE UNIVERSITY WASHINGTON STATE UNIVERSITY OREGON STATE UNIVERSITY UNIVERSITY OF OREGON UNIVERSITY OF WASHINGTON 


\section{Pacific Journal of Mathematics \\ Vol. 87, No. $1 \quad$ January, 1980}

Spiros Argyros, A decomposition of complete Boolean algebras ..........

Gerald A. Beer, The approximation of upper semicontinuous multifunctions

by step multifunctions . . . . ....................

Ehrhard Behrends and Richard Evans, Multiplicity theory for Boolean

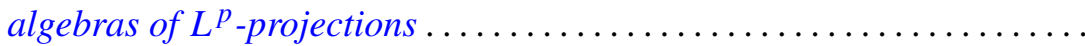

Man-Duen Choi, The full $C^{*}$-algebra of the free group on two

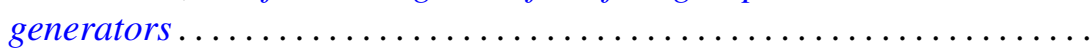

Jen-Chung Chuan, Axioms for closed left ideals in a $C^{*}$-algebra . . . . . . . .

Jo-Ann Deborah Cohen, The strong approximation theorem and locally

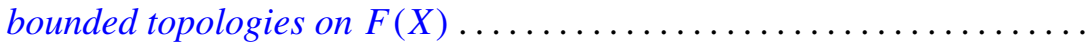

Eugene Harrison Gover and Mark Bernard Ramras, Increasing sequences of Betti numbers............................

Morton Edward Harris, Finite groups having an involution centralizer with

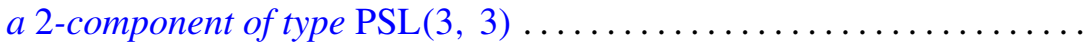

Valéria Botelho de Magalhães Iório, Hopf $C^{*}$-algebras and locally compact

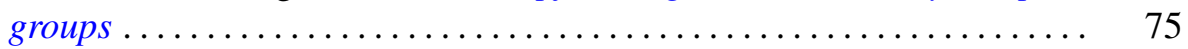

Roy Andrew Johnson, Nearly Borel sets and product measures . . . . . . . . . .

Lowell Edwin Jones, Construction of $Z_{p}$-actions on manifolds . . . . . . . . .

Manuel Lerman and Robert Irving Soare, $d$-simple sets, small sets, and

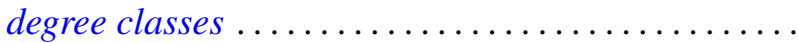

Philip W. McCartney, Neighborly bushes and the Radon-Nikodým property

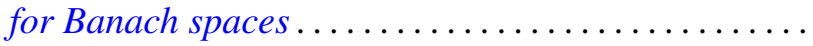

Robert Colman McOwen, Fredholm theory of partial differential equations on complete Riemannian manifolds.

Ernest A. Michael and Carl Preston Pixley, A unified theorem on continuous selections.

Ernest A. Michael, Continuous selections and finite-dimensional sets .

Vassili Nestoridis, Inner functions: noninvariant connected components...

Bun Wong, A maximum principle on Clifford torus and nonexistence of proper holomorphic map from the ball to polydisc.

Steve Wright, Similarity orbits of approximately finite $C^{*}$-algebras . . .

Kenjiro Yanagi, On some fixed point theorems for multivalued

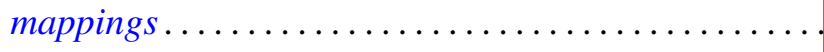

Wieslaw Zelazko, A characterization of LC-nonremovable ideals in commutative Banach algebras 